\title{
The Prevalence Of Binge Eating Disorder And Associated Psychiatric And Substance Use Disorders In A Student Population In Kenya - Towards A Public Health Approach
}

\author{
Dr. Victoria N. Mutiso \\ Africa Mental Health Research and Training Fundation \\ Prof. David M. Ndetei ( $\square$ dmndetei@amhf.or.ke) \\ Africa Mental Health Research and Training Fundation \\ Dr. Esther N. Muia \\ Department of Public and Community Health, Machakos University \\ Rita K. Alietsi \\ Africa Mental Health Research and Training Fundation \\ Lydia Onsinyo \\ Africa Mental Health Research and Training Fundation \\ Dr. Frida Kameti \\ Africa Mental Health Research and Training Fundation \\ Monicah Masake \\ Department of Public and Community Health, Machakos University \\ Dr. Christine Musyimi \\ Africa Mental Health Research and Training Fundation \\ Prof. Daniel Mamah \\ Department of Psychiatry, Washington University Medical School, St. Louis, Missouri
}

\section{Research Article}

Keywords: Eating Disorders, Binge Eating, Bulimia, Co-morbidity, Kenya

Posted Date: February 23rd, 2021

DOI: https://doi.org/10.21203/rs.3.rs-222582/v1

License: @ (i) This work is licensed under a Creative Commons Attribution 4.0 International License. Read Full License 


\section{Abstract}

Background: Changing lifestyles in Kenya can lead to eating related behaviors and problems. The more severe problems are likely to manifest in clinical settings, but the majority and less severe forms will remain unrecognized. There is therefore the need to take a public health awareness approach to identify cases at community level and initiate appropriate intervention. This requires characterization of Eating Disorders (ED) and its associations in the local context. Our focus will be on the more common Binge Eating Disorder (BED). The overarching objective of this study is to generate Kenyan data on BED and fill a gap that exists not only in Kenya but Africa in general. The specific aims are: (1) To document the patterns and prevalence of different symptoms of BED in a student population whose age range represents a significant proportion of the population. (2) To determine associated psychiatric and substance use disorders (3) To determine independent predictors of BED.

Method: We administered to a total of 9742 participants following tools: A researcher designed socio-demographic and economic indicators questionnaire; an instrument on DSM-IV diagnosis of BED and its various symptoms; instruments to determine DSM-IV psychiatric disorders, substance abuse, affectivity, psychosis and stress indicators. The participants were high school, college and university students in four out of the 47 counties in Kenya. We used descriptive and inferential analysis to determine prevalence and association of the different variables. The independent predictors of BED were generated from the generalized linear model $(p<0.05)$.

Results: We found a prevalence of $3.2 \%$ of BED and a wide range of BED symptoms varying from $8.1 \%$ to $19.0 \%$. There were significant ( $p<0.05$ ) associations between BED with various socio-demographic variables and psychiatric and substance use disorders. However, only some of these disorders were independent predictors of BED.

Conclusion: Our findings on prevalence of BED and significant associations with various psychiatric disorders and substance use disorders are similar to those obtained in HICs using similar large scale samples in non-clinical populations. Economic status is not a predictor of BED. Our findings suggest a public health approach to awareness and management.

\section{Background}

\section{Why this study in Kenya}

With increasing standards of living, with the attendant increased availability of food in the Kenyan situation, it can be expected that there will be an increase in eating related problems. The more severe problems can be expected to manifest in clinical settings, with the majority with less severe conditions not likely to find their way to clinical settings. There is therefore the need to take a public health awareness approach to identify cases at community level and initiate appropriate intervention. Most people working in Low and Middle Income Countries (LMIC) are not mental health experts (World Health Organization, 2010) .Yet they work with populations that may have various types of Eating Disorders (ED). It is therefore important to have awareness on ED and any associations in their populations. This requires characterization of ED in the local context. The starting point is to appreciate what is known from studies in other settings. Our focus will be on the more common Binge Eating Disorder (BED).

\section{Eating Disorders Criteria: DSM-IV and DSM-V}

The DSM-V came into existence in 2013 (American Psychiatric Association, 2013). Studies on ED before 2013 used DSM-IV criteria for diagnosis. Studies done using DSM-IV criteria have reported the lifetime prevalence of Anorexia Nervosa (AN), Bulimia Nervosa (BN) and BED to range from $0.3 \%, 0.9 \%$, and $1.6 \%$ respectively (Swanson, Crow, Le Grange, Swendsen, \& Merikangas, 2011) and between 0.7 and $4.3 \%$ in the general population with women being affected approximately 1.5 times more often than men (de Zwaan, 2001).

Using DSM-IV: More focus on clinical population: Studies using DSM-IV criteria on clinical populations of BED have reported associations with mood disorders, substance use, post-traumatic stress disorder (PTSD), suicide (Arcelus, Mitchell, Wales, \& Nielsen, 2011; Krug et al., 2008; Naghavi et al., 2017); anxiety up to 70\% (de Zwaan, 2001; Schwalberg, Barlow, Alger, \& Howard, 1992); elevated prevalence rates of major depression (Ivarsson, Råstam, Wentz, Gillberg, \& Gillberg, 2000; Iwasaki, Matsunaga, Kiriike, Tanaka, \& Matsui, 2000; Lewinsohn, Striegel-Moore, \& Seeley, 2000; Lilenfeld et al., 1997; Woodside et al., 2001); elevated rates of lifetime major depressive disorder (46-58\%), and the presence of axis I psychopathology (de Zwaan, 2001; Specker, de Zwaan, Raymond, \& Mitchell, 1994; Wilfley et al., 2000). BED; obsessive compulsive disorder (OCD) (Hsu, Kaye, \& Weltzin, 1993; Jarry \& Vaccarino, 1996; (McElroy, Phillips, \& Keck Jr, 1994); psychotic disorders (Miotto et al., 2010); (Garfinkel, Garner, Kaplan, Rodin, \& Kennedy, 1983).

Bulik and colleagues (Bulik et al., 2004) reported more depressive and anxiety disorders, cluster B personality disorders, impulsivity and perfectionism personality profiles in women with comorbid alcohol use disorder (AUD) and ED.

Lifetime prevalence rates of SUD have been reported to be 12-18\% in AN and 30-70\% in BN (Bellodi et al., 2001; Herpertz-Dahlmann et al., 2001; Holderness, Brooks-Gunn, \& Warren, 1994; Iwasaki et al., 2000; Lilenfeld et al., 1997; Sullivan, Bulik, Fear, \& Pickering, 1998; Vastag, 2001), compared to an 18\% lifetime prevalence rate of SUD reported in community women (Kessler et al., 1994). All these studies suggest that both BED and SUD share addictive characteristics such as craving, lack of control and denial. Almost a third of women entering treatment for SUD reported binge eating, suggesting that people with BED may use alcohol and other drugs as maladaptive ways to cope with BED (Cohen et al., 2010). Women with PTSD and SUD who reported binge eating behaviors had more severe clinical courses and worse treatment outcomes than those with PTSD and SUD who did not report any binge eating behaviors (Cohen et al., 2010). Substances including alcohol, cocaine, amphetamines, methamphetamine, nicotine and opioids are often used to suppress appetite or increase metabolism, induce vomiting, and self- medicate negative affective mood and anxiety states (Cochrane, Malcolm, \& Brewerton, 1998; Reba-Harrelson et al., 2009; Wolfe \& Maisto, 2000). In the absence of substance use, individuals may use food as an emotional coping response ((Brewerton, 2011). 
Using DSM-V increasing focus on large clinical sample and population studies: More recent studies using DSM-V have generally found higher prevalence of ED and same patterns of associated factors as found in earlier studies using DSM-IV. They have also used large population based samples. In an Australian population (Hay, Girosi, \& Mond, 2015), a study was conducted and merged cross-sectional population survey data of adults aged over 15 years collected in 2008 and 2009 ( $n=6041)$ on ED. They used the criteria based on DSM-V to make diagnosis using data already recorded in the questionnaire. By comparing DSM-IV and DSM-V on the same sample, the prevalence of bulimia nervosa and BED was reduced by nearly $50 \%$ on DSM-IV criteria compared with DSM-V criteria. On DSM-V, the prevalence of BED and sub-threshold BED were 5.6\% - 6.9\% (which was higher than other ED) with a mean age in the 4th to 5th decades compared with a mean age in the 4 th decade for other ED. Thus, they found DSM-V expanded demographic distribution of ED to include adults.

In a report that summarizes clinical European studies and Eating Disorders published between 2015 and first half 2016 (Keski-Rahkonen \& Mustelin, 2016), BED prevalence ranged from $<1-4 \%$ with ED sub-thresholds of $2-3 \%$ of women and $0.3-0.7 \%$ men depending on ED. Over $70 \%$ of individual with different types of ED including BED reported co-morbidity disorders: Anxiety (>40\%), substance use (>10\%), somatic symptoms and increased risk of suicide. Risk factors included: parental psychiatric disorder, prenatal maternal stress, various family factors, childhood overweight and body dissatisfaction in adolescence. They concluded that ED were relatively common, and were often overlooked (only about one third were detected by health care), although they were associated with high co-morbidity and serious health consequences.

In a sample of 36,309 adult participants in a nationally representative sample of adults in the USA, it was found that ED (anorexia nervosa, bulimia nervosa and binge eating disorder (BED)) were associated with lifetime DSM-V mood disorder, anxiety disorder, alcohol, drug use disorders and personality disorder and for BED following somatic disorders: diabetes, hypertension, high cholesterol and triglyceride (Udo \& Grilo, 2019).

In an analysis of 27,111 participants randomly selected from 30,532 children and adolescents in Iran and which did not differentiate between different types of ED found that most common psychiatric disorders were co-morbidities of obsessive-compulsive disorder (20.2\%), agoraphobia (20.2\%), depressive disorder (16.4\%), social phobia (10.1\%), oppositional deficit disorder (3.4\%), generalized anxiety deficit, hyperactivity disorder (7.5\%), conduct disorder (5.7\%), compared to their peers without eating disorder (Meier et al., 2020).

In a USA study using one of the largest samples of 71,712 randomly selected college students from 77 campuses found ED associations with suicidality (ideation and attempts). They found that students with the highest scores of ED had 11 times higher odds of attempting suicide as compared to two times in thresholds symptoms (Lipson \& Sonneville, 2020). They concluded that ED should be a priority within campus suicide prevention efforts. In a systematic review, McDonald et al (McDonald, Rossell, \& Phillipou, 2019) found that individuals with bipolar disorder had increased risk of developing ED compared to the general population, with ED lifetime or current ranging from 1.9-33.3\%. They postulated that ED pathology may occur co-morbidly with bipolar disorder due to shared underlying pathophysiology, with potential implication intervention both pharmacological and psychotherapeutic but his requires more studies.

In a combined systematic review and meta-analysis, Michelle et al (Fornaro et al., 2020) found that in 36 studies that involved 15,084 primary bipolar patients and 11 studies that involved 15,146 people with primary ED found that BED occurred in $12.5 \%$ of bipolar disorders. Overall, bipolar patients with ED were more likely to be females compared with non-ED controls (Fornaro et al., 2020).

The prevalence of co-morbidity PTSD in patients with ED ranges from 9 to $24 \%$, and that co-morbidity PTSD is associated with more severe ED symptoms (Rijkers, Schoorl, van Hoeken, \& Hoek, 2019).

In a meta-analysis study on the rates of co-morbid obsessive compulsive disorder in ED using a binary random effects model, Mandelli et al (Mandelli, Draghetti, Albert, De Ronchi, \& Atti, 2020) found that globally, $18 \%$ and $15 \%$ of all patients with ED had a lifetime and current co-morbidity with OCD in clinical populations respectively, using DSM-V criteria. They concluded that OCD co-morbidity of ED affects almost $20 \%$ of patients in cross-sectional observations and up to nearly $40 \%$ in prospective follow up studies and therefore necessary to consider OCD in order to arrive at a better diagnostic and prognostic arriving and targeted treatment.

From the available literature, nearly all the studies have been reported from High Income Countries (HIC) Western countries and one from Iran. In particular, no studies have been done in Kenya relating to community prevalence of ED, the different symptoms of BED, associated psychiatric conditions including psychosis and SUD and what independently predicts BED. The overall objective of this study is to fill that gap not only for Kenya but for Africa in general.

The specific aims are:

1. To document the patterns and prevalence of different symptoms of BED in a student population that represents significant properties of Kenyan population.

2. To determine associated psychiatric and substance use disorders including symptoms of affective and psychotic disorders.

3. To determine independent predictors of BED.

4. To use the findings of the study to suggest a strategy for a public health intervention that is context appropriate.

\section{Methods}

\section{Recruitment and Data Collection}

The study was a non-clinical population based cross sectional study. Permission was sought from Institutional heads for the university and college students while for school going children in the community, permission was sought from the local administration (the schools were closed). Participants were recruited from Nairobi and three other counties in South Eastern Kenya - namely Machakos, Kitui and Makueni Counties in Kenya. University and college students were approached after lecture hours in their classrooms. The research assistants were informed on the schedule for the different classes as they appeared in the

Page $3 / 16$ 
timetables each day of the week. The high school students were directed to specific public meeting areas for assessment with the help of the local community leaders. Participants were only included in the study if they met the requirements i.e. were in high school, college or university, had voluntarily agreed to participate in the study. Informed consent was obtained from participants aged $18+$ and from parents of participants that were below the age of 18 and also assented. The age range for high school students is 14-18 years. For college/university the age range starts 19-25 for most students but also have late entry students. High school, college and university students in Kenya are all fluent in English - English is a national language and the official language for all communications and medium of learning. All participants, regardless of their age and provided they had been officially registered as students were included and treated as students. A self-administered questionnaire was used to collect data from participants. A total of 9,742 participants from different years of study and courses were recruited for the study. As part of preparation for this study, we discussed with institutions on the need to incorporate mental health in their institutional health services for their students in case the awareness of mental disorders during the study prompted students to seek for mental health services. As for the high school students, we had trained staff at the local health center facility on the WHO mhGAP-IG. We also informed and directed the participants where to seek help at their institutions and community levels in case they needed any help. All the participants who were approached participated.

\section{Tools}

1. Socio-demographic characteristics: A researcher-designed questionnaire was used to get the socio-demographics information of the respondents. Sociodemographic variables included age, gender, highest level of education, marital status and birth order.

2. Economic Indicators: The respondents completed questions regarding household items, water source, toilet type and cooking method. These were used to estimate socio-economic status by creation of wealth index (Smits \& Steendijk, 2015). The wealth index used is based on the World Bank

Recommendation for LMICs(Smits \& Steendijk, 2015) and has been adopted by the Kenya Government for use in Kenya. It is classified into five sections, quintile 1-5 with quintile 1 representing the lowest level of wealth and 5 the highest level.

3. Psychiatric conditions: The psychiatric diagnostic screening questionnaire (PDSQ) was used to assess psychiatric conditions of the respondents. It consists of 126 questions assessing the symptoms of 13 DSM-IV Axis 1 disorders: mood disorders (major depressive disorder [MDD]); anxiety disorders (panic disorder, agoraphobia, PTSD, obsessive-compulsive disorder, generalized anxiety disorder [GAD], and social phobia); substance use disorders (alcohol abuse/dependence and drug abuse/dependence); and somatoform disorders (somatization disorder and hypochondriasis). In addition, it has a 6-item psychosis screen. For each psychiatric disorder, there are several questions which are computed to arrive at a diagnosis. The disorders chosen for coverage were selected because they are the most prevalent in epidemiological surveys of the community (Kessler et al., 1994; Robins, 1991) and the most frequently reported in large clinical samples (Koenigsberg, Kaplan, Gilmore, \& Cooper, 1985; Mezzich, Fabrega, Coffman, \& Haley, 1989; Shear et al., 2000). In a validity study in which 994 psychiatric outpatients completed the scale (Zimmerman \& Mattia, 2001), the 13 PDSQ subscales demonstrated good to excellent levels of internal consistency. Cronbach's a was greater than .80 for 12 of the 13 subscales, and the mean of the a coefficients was .86. Test-retest reliability was examined in the 185 subjects who completed the PDSQ 2 times less than a week apart. Test-retest reliability coefficients were greater than 0.80 for 9 subscales, and the mean of the test-retest correlation coefficients was 0.83 . The convergent and discriminant validity of the PDSQ subscales(Campbell \& Fiske, 1959) was examined in 361 patients who completed a package of questionnaires at home less than a week after completing the PDSQ. The last six questions from PDSQ on major depressive episode domain are used to measure suicidal ideation classified as follows: frequently think of dying in passive ways like going to sleep and not waking up, wishing to be dead, thinking you were better off dead, having thoughts of suicide, seriously considering taking life, and thinking about specific ways of taking your life. Further, PDSQ has 10 questions on BED each question asking about specific symptoms of BED. The 10 questions are computed to make a diagnosis of BED. The questions were coded as No or Yes with No having a value of zero and Yes having a value of one.

4. Psychosis, affectivity. The Washington Early Recognition Center Affectivity and Psychosis (WERCAP) screen was used to quantitatively assess psychosis-risk symptoms and bipolar-risk symptoms (affectivity) based on the frequency of symptoms and their effects on functioning(Mamah, Owoso, Sheffield, \& Bayer, 2014). It has high test-retest reliability and validity, with affectivity of sensitivity of .91 , specificity of .71 , psychosis sensitivity .88 and specificity of .82(Mamah et al., 2014).

5. Drug and substance: The WHO's Alcohol, Smoking, and Substance Involvement Screening Test (ASSIST) (Humeniuk et al., 2010) was used only to determine the prevalence of different types of substance use on a Yes or No dimension.

\section{Data management and Analysis}

The coded data was checked, cleaned and exported into Statistical Package for the Social Sciences (SPSS) version 23.0 for analysis. Basic descriptive statistics were carried out to estimate the prevalence of Bulimia/binge eating disorder as well as the participant's socio-demographic and socio-economic characteristics. Scores were grouped into two: those with the eating disorder and those without the eating disorder. Estimation of univariate associations between Bulimia/binge eating disorder and other variables was carried out by fitting bivariate logistic regression that was also used to identify confounding factors. Variables with p-value less than 0.05 were then fitted into generalized linear model, with logit as the link, to identify independent predictors of Bulimia/Binge eating disorder. The strength and significance of the association between the variables and Bulimia/Binge eating disorder was assessed by calculating the adjusted odds ratio with a $95 \%$ confidence level. Correlation analysis was also carried out between Bulimia/Binge eating disorder and the psychiatric conditions. All the tests carried out were two-sided with a set P-value of less than $0.05(\mathrm{p}<0.05)$.

All methods were carried out in accordance with relevant guidelines and regulations.

\section{Results}

\section{Socio-Demographic Characteristics of Respondents}


Table 1 presents the results of socio-demographic characteristics of the respondents. Males (53.5\%) were more than females. Mean age was 21.4 , median 21.3 (range 15-43) years. Majority of the respondents were single (93.4\%), mainly from university (68.6\%), most (56.9\%) were either first or second born in their families. The wealth index was evenly distributed among the first 5 quintiles with the fifth quintile having the lowest proportion (16.6\%).

Table 1

Socio-Demographic and economic factors of Respondents

\begin{tabular}{|c|c|c|c|}
\hline Variable & Category & Frequency $(\mathrm{N}=9742)$ & Percentage (\%) \\
\hline \multirow[t]{3}{*}{ Gender } & Male & 5173 & 53.5 \\
\hline & Female & 4500 & 46.5 \\
\hline & Missing & 69 & 0.7 \\
\hline Age & Mean; Median; SD; Range & $21.4 ; 21.3 ; 2.4 ; 15-43$ & \\
\hline \multirow[t]{4}{*}{ Marital status } & Married & 607 & 6.3 \\
\hline & Single & 9057 & 93.4 \\
\hline & Others & 38 & 0.4 \\
\hline & Missing & 40 & 0.4 \\
\hline \multirow[t]{5}{*}{ Religion } & Protestant & 5512 & 57.1 \\
\hline & Catholic & 3359 & 34.8 \\
\hline & Muslim & 410 & 4.2 \\
\hline & Other & 368 & 3.8 \\
\hline & Missing & 93 & 1.0 \\
\hline \multirow[t]{4}{*}{ Birth order } & $1-2$ & 5539 & 56.9 \\
\hline & $3-5$ & 3271 & 33.6 \\
\hline & $6+$ & 920 & 9.5 \\
\hline & Missing & 12 & 0.1 \\
\hline \multirow[t]{4}{*}{ Level of Education } & High School & 1506 & 15.5 \\
\hline & College & 1534 & 15.8 \\
\hline & University & 6648 & 68.6 \\
\hline & Missing & 54 & 0.6 \\
\hline \multirow{5}{*}{$\begin{array}{l}\text { Wealth Index } \\
\text { (Quintile1 = Lowest; } \\
\text { Quintile } 5 \text { = Highest) }\end{array}$} & Quintile 1 & 2044 & 21.0 \\
\hline & Quintile 2 & 1865 & 19.1 \\
\hline & Quintile 3 & 2002 & 20.6 \\
\hline & Quintile 4 & 2214 & 22.7 \\
\hline & Quintile 5 & 1617 & 16.6 \\
\hline
\end{tabular}

\section{Prevalence of Symptoms of Bulimia/Binge Eating Disorder}

Figure 1 presents a pictorial representation in descending order of BED symptoms using 10 PDSQ items used to screen for BED.

\section{Associated Factors}

\section{i. Socio-demographic factors and wealth index}

Table 2 summarizes the socio-demographic factors associated with BED at bivariate level. The younger age group, representing high school students were 1.39 times more likely to have Bulimia/Binge Eating Disorder as compared to those in the university $(p<0.05)$. No other socio demographic factors or level of wealth index was associated with BED. 
Table 2

Socio-demographic factors associated with BULIMIA/BINGE EATING DISORDER

\begin{tabular}{|c|c|c|c|c|c|}
\hline \multirow[t]{2}{*}{ Variable } & \multirow[t]{2}{*}{ Category } & \multicolumn{2}{|c|}{$\begin{array}{l}\text { Bulimia/Binge } \\
\text { Eating Disorder }\end{array}$} & \multirow[t]{2}{*}{ O.R (95\% C.I.) } & \multirow[t]{2}{*}{ Sig. } \\
\hline & & No & Yes & & \\
\hline \multirow[t]{2}{*}{ Gender } & Male & $5005(96.8 \%)$ & $167(3.2 \%)$ & $1.00(0.80-1.26)$ & 0.987 \\
\hline & Female & $4354(96.8 \%)$ & $145(3.2 \%)$ & Ref. & \\
\hline Age & Mean \pm SD & $21.4 \pm 2.4$ & $21.1 \pm 2.5$ & $1.01(0.97-1.06)$ & 0.619 \\
\hline \multirow[t]{3}{*}{ Marital status } & Married & $589(97.0 \%)$ & $18(3.0 \%)$ & $0.36(0.10-1.27)$ & 0.111 \\
\hline & Single & $8765(96.8 \%)$ & $290(3.2 \%)$ & $0.39(0.12-1.26)$ & 0.115 \\
\hline & Others & $35(92.1 \%)$ & $3(7.9 \%)$ & Ref. & \\
\hline \multirow[t]{4}{*}{ Religion } & Protestant & $5353(97.1 \%)$ & $158(2.9 \%)$ & $0.96(0.52-1.78)$ & 0.892 \\
\hline & Catholic & $3223(96.0 \%)$ & $135(4.0 \%)$ & $1.36(0.73-2.54)$ & 0.335 \\
\hline & Muslim & 401(97.8\%) & $9(2.2 \%)$ & $0.73(0.30-1.78)$ & 0.486 \\
\hline & Other & $357(97.0 \%)$ & $11(3.0 \%)$ & Ref. & \\
\hline \multirow[t]{3}{*}{ Birth order } & $1-2$ & $5343(96.5 \%)$ & 194(3.5\%) & $1.12(0.75-1.66)$ & 0.589 \\
\hline & $3-5$ & $3178(97.2 \%)$ & $93(2.8 \%)$ & $0.90(0.59-1.37)$ & 0.622 \\
\hline & $6+$ & 891(96.8\%) & $29(3.2 \%)$ & Ref. & \\
\hline \multirow[t]{3}{*}{ Level of Education } & High School & 1442(95.8\%) & $63(4.2 \%)$ & $1.39(1.04-1.85)$ & 0.026 \\
\hline & College & 1487(96.9\%) & $47(3.1 \%)$ & $1.00(0.73-1.38)$ & 0.984 \\
\hline & University & 6444(96.9\%) & $203(3.1 \%)$ & Ref. & \\
\hline \multirow[t]{5}{*}{ Wealth Index } & Quintile 1 & 1891(97.3\%) & $53(2.7 \%)$ & $0.82(0.56-1.18)$ & 0.282 \\
\hline & Quintile 2 & 1886(97.1\%) & $57(2.9 \%)$ & $0.88(0.61-1.27)$ & 0.491 \\
\hline & Quintile 3 & 1833(96.4\%) & $69(3.6 \%)$ & $1.10(0.78-1.55)$ & 0.602 \\
\hline & Quintile 4 & $1879(96.4 \%)$ & $71(3.6 \%)$ & $1.10(0.78-1.55)$ & 0.585 \\
\hline & Quintile 5 & 1864(96.7\%) & $64(3.3 \%)$ & Ref. & \\
\hline
\end{tabular}

\section{ii. Psychiatric Disorders}

Table 3 presents the results on the psychiatric disorders associated with BED at bivariate level. There was significant association between all psychiatric disorders and BED $(p<0.001)$. 
Table 3

Psychiatric Disorders associated with BULIMIA/BINGE EATING DISORDER

\begin{tabular}{|c|c|c|c|c|c|}
\hline \multirow[t]{3}{*}{ Condition } & \multirow[t]{3}{*}{ Category } & \multirow{2}{*}{\multicolumn{2}{|c|}{$\begin{array}{l}\text { Bulimia/Binge } \\
\text { Eating Disorder Score: }\end{array}$}} & \multirow[t]{3}{*}{ O.R (95\% C.I.) } & \multirow[t]{3}{*}{ Sig. } \\
\hline & & & & & \\
\hline & & No & Yes & & \\
\hline \multirow[t]{2}{*}{ Major Depressive Disorder Score: } & No & $7608(98.8 \%)$ & $92(1.2 \%)$ & Ref. & \\
\hline & Yes & $1816(89.0 \%)$ & $224(11.0 \%)$ & $10.20(7.96-13.07)$ & $<0.001$ \\
\hline \multirow[t]{2}{*}{ PTSD Score: } & No & $6973(98.4 \%)$ & $115(1.6 \%)$ & Ref. & \\
\hline & Yes & $2451(92.4 \%)$ & $201(7.6 \%)$ & $4.97(3.94-6.28)$ & $<0.001$ \\
\hline \multirow[t]{2}{*}{ Obsessive Compulsive Disorder Score: } & No & $3411(99.4 \%)$ & $21(0.6 \%)$ & Ref. & \\
\hline & Yes & 6013(95.3\%) & $295(4.7 \%)$ & $7.97(5.11-12.43)$ & $<0.001$ \\
\hline \multirow[t]{2}{*}{ Panic Disorder Score: } & No & 7735(98.6\%) & $110(1.4 \%)$ & Ref. & \\
\hline & Yes & 1688(89.1\%) & $206(10.9 \%)$ & $8.58(6.77-10.88)$ & $<0.001$ \\
\hline \multirow[t]{2}{*}{ Psychosis Score: } & No & $5644(99.0 \%)$ & $56(1.0 \%)$ & Ref. & \\
\hline & Yes & $3780(93.6 \%)$ & $260(6.4 \%)$ & $6.93(5.18-9.28)$ & $<0.001$ \\
\hline \multirow[t]{2}{*}{ Agoraphobia Score: } & No & $6321(98.9 \%)$ & $68(1.1 \%)$ & Ref. & \\
\hline & Yes & $3102(92.6 \%)$ & $248(7.4 \%)$ & $7.43(5.66-9.75)$ & $<0.001$ \\
\hline \multirow[t]{2}{*}{ Social Phobia Score: } & No & $4802(98.9 \%)$ & $54(1.1 \%)$ & Ref. & \\
\hline & Yes & $4622(94.6 \%)$ & $262(5.4 \%)$ & $4.70(3.74-5.90)$ & $<0.001$ \\
\hline \multirow[t]{2}{*}{ Alcohol Abuse/Dependence Score: } & No & 7395(98.1\%) & $147(1.9 \%)$ & Ref. & \\
\hline & Yes & $2029(92.3 \%)$ & $169(7.7 \%)$ & $4.19(3.34-5.25)$ & $<0.001$ \\
\hline \multirow[t]{2}{*}{ Drug Abuse/Dependence Score: } & No & 7904(97.9\%) & $166(2.1 \%)$ & Ref. & \\
\hline & Yes & $1520(91.0 \%)$ & $150(9.0 \%)$ & $5.90(4.69-7.43)$ & $<0.001$ \\
\hline \multirow[t]{2}{*}{ Generalized Anxiety Disorder Score: } & No & $8266(97.9 \%)$ & $173(2.1 \%)$ & Ref. & \\
\hline & Yes & $1158(89.0 \%)$ & $143(11.0 \%)$ & $4.27(3.40-5.36)$ & $<0.001$ \\
\hline \multirow[t]{2}{*}{ Somatization Disorder Score: } & No & $7104(98.2 \%)$ & $132(1.8 \%)$ & Ref. & \\
\hline & Yes & $2320(92.7 \%)$ & $184(7.3 \%)$ & $10.20(7.96-13.07)$ & $<0.001$ \\
\hline \multirow[t]{2}{*}{ Hypochondriasis Score: } & No & 6972(98.3\%) & $122(1.7 \%)$ & Ref. & \\
\hline & Yes & $2452(92.7 \%)$ & 194(7.3\%) & $4.52(3.59-5.70)$ & $<0.001$ \\
\hline \multirow[t]{2}{*}{ Suicidality } & No & $7416(98.4 \%)$ & $120(1.6 \%)$ & Ref. & \\
\hline & Yes & 2008(91.1\%) & $196(8.9 \%)$ & $6.03(4.78-7.61)$ & $<0.001$ \\
\hline Sum Score of WERC Stress Screen: & Mean $\pm S D$ & $25.2 \pm 26.4$ & $49.9 \pm 38.4$ & $1.02(1.02-1.02)$ & $<0.001$ \\
\hline Total sum of WERCAP Affectivity & Mean $\pm S D$ & $10.2 \pm 8.3$ & $17.2 \pm 9.8$ & $1.09(1.07-1.10)$ & $<0.001$ \\
\hline Total sum of WERCAP Psychosis: & Mean \pm SD; & $8.4 \pm 9.6$ & $18.2 \pm 13.1$ & $1.07(1.06-1.08)$ & $<0.001$ \\
\hline
\end{tabular}

The correlation between BED and psychiatric disorders was highly significant $(p<0.001)$ for all psychiatric disorders (Table 4). 
Table 4

Correlation between BULIMIA/BINGE EATING DISORDER scores and psychiatric conditions scores

\begin{tabular}{|c|c|c|c|c|c|c|c|c|c|c|c|c|c|c|c|}
\hline $\begin{array}{l}\text { Pearson } \\
\text { Correlation }\end{array}$ & 1 & 2 & 3 & 4 & 5 & 6 & 7 & 8 & 9 & 10 & 11 & 12 & 13 & 14 & 15 \\
\hline $\begin{array}{l}\text { 1. Bulimia/Binge } \\
\text { Eating Disorder }\end{array}$ & 1 & & & & & & & & & & & & & & \\
\hline $\begin{array}{l}\text { 2. Major } \\
\text { Depressive } \\
\text { Disorder Score: }\end{array}$ & $.488^{\star \star}$ & 1 & & & & & & & & & & & & & \\
\hline 3. PTSD Score: & $.412^{\star \star}$ & $.514^{\star *}$ & 1 & & & & & & & & & & & & \\
\hline $\begin{array}{l}\text { 4. Obsessive } \\
\text { Compulsive } \\
\text { Disorder. }\end{array}$ & $.377^{* \star}$ & $.426^{\star *}$ & $.391^{\star *}$ & 1 & & & & & & & & & & & \\
\hline $\begin{array}{l}\text { 5. Panic Disorder } \\
\text { Score: }\end{array}$ & $.442^{\star *}$ & $.497^{\star \star}$ & $.449^{\star *}$ & $.526^{\star *}$ & 1 & & & & & & & & & & \\
\hline 6. Psychosis Score: & $.466^{\star *}$ & $.467^{\star \star}$ & $.443^{\star *}$ & $.476^{\star *}$ & $.563^{\star \star}$ & 1 & & & & & & & & & \\
\hline $\begin{array}{l}\text { 7. Agoraphobia } \\
\text { Score: }\end{array}$ & $.396^{* *}$ & $.432^{\star *}$ & $.389^{\star *}$ & $.461^{\star *}$ & $.502^{\star \star}$ & $.472^{\star \star}$ & 1 & & & & & & & & \\
\hline $\begin{array}{l}\text { 8. Social Phobia } \\
\text { Score: }\end{array}$ & $.363^{\star *}$ & $.462^{\star \star}$ & $.376^{\star *}$ & $.469^{\star *}$ & $.453^{\star \star}$ & $.425^{\star \star}$ & $.573^{\star *}$ & 1 & & & & & & & \\
\hline $\begin{array}{l}\text { 9. Alcohol } \\
\text { Abuse/Dependence }\end{array}$ & $.380^{\star *}$ & $.316^{\star *}$ & $.269^{\star *}$ & $.211^{\star *}$ & $.298^{\star \star}$ & $.334^{\star \star}$ & $.284^{\star *}$ & $.275^{\star *}$ & 1 & & & & & & \\
\hline $\begin{array}{l}\text { 10. Drug } \\
\text { Abuse/Dependence }\end{array}$ & $.371^{\star *}$ & $.295^{\star \star}$ & $.267^{\star *}$ & $.199^{\star *}$ & $.258^{\star *}$ & $.312^{\star *}$ & $.247^{\star \star}$ & $.233^{\star *}$ & $.617^{\star \star}$ & 1 & & & & & \\
\hline $\begin{array}{l}\text { 11. Generalized } \\
\text { Anxiety Disorder } \\
\text { Score: }\end{array}$ & $.376^{\star *}$ & $.519^{\star \star}$ & $.413^{\star \star}$ & $.425^{\star *}$ & $.486^{\star *}$ & $.424^{\star *}$ & $.468^{\star *}$ & $.556^{\star *}$ & $.275^{\star *}$ & $.276^{\star *}$ & 1 & & & & \\
\hline $\begin{array}{l}\text { 12. Somatization } \\
\text { Disorder Score: }\end{array}$ & $.344^{* *}$ & $.411^{\star \star}$ & $.333^{\star \star}$ & $.301^{\star *}$ & $.391^{\star *}$ & $.354^{\star *}$ & $.377^{\star *}$ & $.377^{\star \star}$ & $.298^{\star *}$ & $.290^{\star *}$ & $.424^{\star *}$ & 1 & & & \\
\hline $\begin{array}{l}13 . \\
\text { Hypochondriasis } \\
\text { Score: }\end{array}$ & $.350^{\star *}$ & $.397^{\star \star}$ & $.339^{\star *}$ & $.304^{\star *}$ & $.424^{\star *}$ & $.383^{\star *}$ & $.376^{\star *}$ & $.365^{\star *}$ & $.305^{\star *}$ & $.314^{\star *}$ & $.451^{\star *}$ & $.525^{\star *}$ & 1 & & \\
\hline $\begin{array}{l}\text { 14. WERC Stress } \\
\text { Screen score. }\end{array}$ & $.308^{\star *}$ & $.438^{\star \star}$ & $.362^{\star *}$ & $.311^{\star \star}$ & $.339^{\star *}$ & $.299^{\star \star}$ & $.300^{\star *}$ & $.311^{* *}$ & $.217^{\star \star}$ & $.211^{\star \star}$ & $.345^{\star \star}$ & $.275^{\star *}$ & $.280^{\star \star}$ & 1 & \\
\hline $\begin{array}{l}\text { 15. Total sum of } \\
\text { WERCAP } \\
\text { Affectivity } \\
\text { Disorder: }\end{array}$ & $.302^{* *}$ & $.504^{\star *}$ & $.348^{\star *}$ & $.322^{\star *}$ & $.338^{\star *}$ & $.329^{\star *}$ & $.303^{\star \star}$ & $.334^{\star *}$ & $.183^{* *}$ & $.178^{\star \star}$ & $.409^{\star \star}$ & $.294^{\star *}$ & $.276^{\star \star}$ & $.415^{\star \star}$ & 1 \\
\hline $\begin{array}{l}\text { 16. Total sum of } \\
\text { WERCAP Psychosi: }\end{array}$ & $.353^{\star *}$ & $.487^{\star *}$ & $.382^{\star *}$ & $.353^{\star *}$ & $.393^{\star *}$ & $.445^{\star *}$ & $.339^{\star *}$ & $.327^{\star *}$ & $.242^{\star \star}$ & $.231^{\star *}$ & $.386^{\star *}$ & $.291^{\star \star}$ & $.316^{\star \star}$ & $.418^{\star *}$ & $.66 \mathrm{C}$ \\
\hline
\end{tabular}

iii. Drug and substance use

Table 5 presents the results on drug/substances associated with BED at bivariate level. There was significant association between all the substances and BED except for alcohol $(p<0.05)$. The prevalence of SUD in BED varied from 3.7-7.6\%. Alcohol had the lowest prevalence of $3.7 \%$. 
Table 5

Drug/Substances associated with Bulimia/Binge eating disorder.

\begin{tabular}{|c|c|c|c|c|c|}
\hline \multirow[t]{3}{*}{ Substance } & \multirow[t]{3}{*}{ Category } & \multirow{2}{*}{\multicolumn{2}{|c|}{$\begin{array}{l}\text { Bulimia/Binge } \\
\text { Eating Disorder Score: }\end{array}$}} & \multirow[t]{3}{*}{ O.R (95\% C.I.) } & \multirow[t]{3}{*}{ Sig. } \\
\hline & & & & & \\
\hline & & No & Yes & & \\
\hline \multirow[t]{2}{*}{ Tobacco } & No & 8930(96.9\%) & $287(3.1 \%)$ & Ref & \\
\hline & Yes & $494(94.5 \%)$ & $29(5.5 \%)$ & $0.55(0.37-0.81)$ & 0.003 \\
\hline \multirow[t]{2}{*}{ Alcohol } & No & $7768(96.9 \%)$ & $252(3.1 \%)$ & Ref & \\
\hline & Yes & 1656(96.3\%) & $64(3.7 \%)$ & $1.19(0.90-1.58)$ & 0.219 \\
\hline \multirow[t]{2}{*}{ Cannabis } & No & $8981(96.9 \%)$ & $292(3.1 \%)$ & Ref & \\
\hline & Yes & $443(94.9 \%)$ & $24(5.1 \%)$ & $1.67(1.09-2.55)$ & 0.019 \\
\hline \multirow[t]{2}{*}{ Sedatives } & No & $9266(96.8 \%)$ & $303(3.2 \%)$ & Ref & \\
\hline & Yes & $158(92.4 \%)$ & $13(7.6 \%)$ & $2.52(1.41-4.48)$ & 0.002 \\
\hline \multirow[t]{2}{*}{ Khat/Amphetamine } & No & $9151(96.8 \%)$ & $298(3.2 \%)$ & Ref & \\
\hline & Yes & $273(93.8 \%)$ & $18(6.2 \%)$ & $2.02(1.24-3.31)$ & 0.005 \\
\hline
\end{tabular}

iv. Independent predictors of ED

Tables 6 summarizes the independent predictors of BED using AOR analysis. Major depressive disorder, OCD, panic disorder, psychosis, agoraphobia, drug abuse, generalized anxiety disorder, suicidality and WERCAP stress were the leading independent predictors of BED. 
Table 6

Independent Predictors of ED

\begin{tabular}{|c|c|c|c|c|c|}
\hline & \multirow[t]{2}{*}{ Category } & \multirow[t]{2}{*}{ A.O.R. } & \multicolumn{2}{|c|}{ 95\% C.I A.O.R. } & \multirow[t]{2}{*}{ Sig. } \\
\hline & & & Lower & Upper & \\
\hline \multirow[t]{3}{*}{ Level of Education } & University & 1.011 & 0.738 & 1.386 & 0.943 \\
\hline & College & 0.804 & 0.531 & 1.218 & 0.304 \\
\hline & High School & Ref & & & \\
\hline \multirow[t]{2}{*}{ Tobacco } & Yes & 0.986 & 0.605 & 1.606 & 0.955 \\
\hline & No & Ref & & & \\
\hline \multirow[t]{2}{*}{ Cannabis } & Yes & 0.906 & 0.527 & 1.560 & 0.723 \\
\hline & No & Ref & & & \\
\hline \multirow[t]{2}{*}{ Sedatives } & Yes & 1.059 & 0.547 & 2.048 & 0.866 \\
\hline & No & Ref & & & \\
\hline \multirow[t]{2}{*}{ Khat/amphetamine } & Yes & 1.231 & 0.691 & 2.192 & 0.481 \\
\hline & No & Ref & & & \\
\hline \multirow[t]{2}{*}{ Major Depressive Disorder Score: } & Yes & 2.478 & 1.807 & 3.399 & $<0.001$ \\
\hline & No & Ref & & & \\
\hline \multirow[t]{2}{*}{ PTSD Score: } & Yes & 1.039 & 0.785 & 1.377 & 0.788 \\
\hline & No & Ref & & & \\
\hline \multirow[t]{2}{*}{ Obsessive Compulsive Disorder Score: } & Yes & 1.810 & 1.105 & 2.965 & 0.018 \\
\hline & No & Ref & & & \\
\hline \multirow[t]{2}{*}{ Panic Disorder Score: } & Yes & 2.059 & 1.548 & 2.740 & $<0.001$ \\
\hline & No & Ref & & & \\
\hline \multirow[t]{2}{*}{ Psychosis Score: } & Yes & 1.406 & 0.988 & 2.000 & 0.058 \\
\hline & No & Ref & & & \\
\hline \multirow[t]{2}{*}{ Agoraphobia Score: } & Yes & 1.959 & 1.409 & 2.724 & $<0.001$ \\
\hline & No & Ref & & & \\
\hline \multirow[t]{2}{*}{ Social Phobia Score: } & Yes & 0.879 & 0.612 & 1.263 & 0.487 \\
\hline & No & Ref & & & \\
\hline \multirow[t]{2}{*}{ Alcohol Abuse/Dependence Score: } & Yes & 1.236 & 0.895 & 1.708 & 0.198 \\
\hline & No & Ref & & & \\
\hline \multirow[t]{2}{*}{ Drug Abuse/Dependence Score: } & Yes & 1.552 & 1.122 & 2.147 & 0.008 \\
\hline & No & Ref & & & \\
\hline \multirow[t]{2}{*}{ Generalized Anxiety Disorder Score: } & Yes & 1.681 & 1.277 & 2.213 & $<0.001$ \\
\hline & No & Ref & & & \\
\hline \multirow[t]{2}{*}{ Somatization Disorder Score: } & Yes & 1.216 & 0.925 & 1.598 & 0.161 \\
\hline & No & Ref & & & \\
\hline \multirow[t]{2}{*}{ Hypochondriasis Score: } & Yes & 0.891 & 0.664 & 1.195 & 0.442 \\
\hline & No & Ref & & & \\
\hline \multirow[t]{2}{*}{ Suicidality } & Yes & 1.639 & 1.243 & 2.161 & $<0.001$ \\
\hline & No & Ref & & & \\
\hline \multicolumn{2}{|l|}{ Total sum of WERCAP Affectivity: } & 1.004 & 0.986 & 1.022 & 0.660 \\
\hline \multicolumn{2}{|l|}{ Total sum of WERCAP Psychosis: } & 1.009 & 0.996 & 1.023 & 0.177 \\
\hline
\end{tabular}

Note; Ref-Reference Category; C.I.-Confidence Interval; n/s-Not significant; A.O.R.- Adjusted Odds Ratio

Page 10/16 


\begin{tabular}{|c|c|c|c|c|c|}
\hline & \multirow[t]{2}{*}{ Category } & \multirow[t]{2}{*}{ A.O.R. } & \multicolumn{2}{|c|}{ 95\% C.I A.O.R. } & \multirow[t]{2}{*}{ Sig. } \\
\hline & & & Lower & Upper & \\
\hline Sum Score of WERC Stress Screen: & & 1.005 & 1.001 & 1.008 & 0.022 \\
\hline
\end{tabular}

\section{Discussion}

We present the first African reported non-clinical population epidemiological patterns of different symptoms in BED to be reported specifically in a wide range of students with a large sample as opposed to clinical epidemiological patterns that are based on clinical populations. To the best of our knowledge, it is one of the two reported studies on college students globally (the other one was done in USA college students) and one of the few large sample non-clinical populations studies reported in global literature. It is also one of the two large sample reported globally to investigate ED across a wide age range, starting from 15 years to adulthood reported by Phillipa et al (Hay et al., 2015) in Australia.

\section{Response rate}

The $100 \%$ response rate in our study is not unique and is common in Kenyan community-based mental health related and student surveys (Ndetei, Khasakhala, Mutiso, Ongecha-Owuor, \& Kokonya, 2009, 2010). Students and parents place immense value on such surveys, as education is regarded as the best investment, with the highest potential to propel students into successful futures and help them and their families escape from poverty. However, we point out that we approached colleges and university students at specific time points as a "captive" group, which may have contributed to the high response rate. The high school students voluntarily came to the community centers, which means they were already motivated to participate.

\section{The Socio-Demographics}

Males were more than the females for the following two plausible reasons. Most of the college students were in technical colleges that are patronized by male than female gender. Secondly, the data collection for high school students was done when the schools were closed. Boys more than girls were more likely to be allowed to go to the data collection site. The wide range in age is easily explained by late age entry to schools, colleges and university. Since most of the participants were students, it is not surprising that most were single. Other types of marriages were come we stay arrangement common in college and university students as one of the ways of sharing accommodation costs.

Kenyan family structure is increasingly getting smaller, explaining 1st and 2nd birth order (Kenya National Bureau of Statistics (KNBS), 2019). We did not access as many high school students as we did in the captive groups that we found in college and university students since the data was collected when the schools were closed. There was an equitable distribution of wealth index but it was less for quintile 5 as compared with the rest. This is most likely a reflection of wealthy parents taking children to private institutions which were not represented in our study. Our findings suggest that BED is not a reflection of socioeconomic status, even when this socio-economic status may reflect relative availability of food.

\section{Prevalence of BED}

The overall prevalence of BED using DSM-IV criteria (3.2\%) found in our study is much higher than the "estimated" general population lifetime prevalence of BED of $0.3-1.6 \%$ reported by Swanson et al (Swanson et al., 2011) using DSM-IV criteria but within the range of $0.7-4.3 \%$ reported by American Psychiatric Association (APA)(American Psychiatric Association, 2013), using the DSM-V. If we apply the finding of Philippa et al (Hay et al., 2015) in Australia that DSM-V expanded ED by $50 \%$ over DSM-IV, which was used in this study, then the $3.2 \%$ found in this study could be increased by $50 \%$ to a prevalence of $4.8 \%$. This is just above the $4.3 \%$ upper level reported by APA using DSM-V criteria (American Psychiatric Association, 2013). These findings are essentially identical. Even much higher are the prevalence of individual BED symptoms. Our sample was drawn from student populations as opposed to the general population. It is possible student population in institutions are different from general population and therefore cannot extrapolate this to the general population. However, in favor of plausible extrapolation to the general populations is that these students were drawn from the general population. Given the pyramid structure of the Kenyan population (Kenya National Bureau of Statistics (KNBS), 2019), these students represent a significant proportion of the total Kenyan population.

We find our results surprising that BED could be $3.2 \%$ and possibly $4.8 \%$ if we had used DSM-V and similar to WPA findings, coming from LMIC setting, often associated with limited resources and often reported malnutrition or shortage of food (Grace, Davenport, Funk, \& Lerner, 2012).

We could not find any study anywhere that reported the prevalence of individual symptoms to compare with our findings of $19.0-8.1 \%$ of the various items. These prevalence of individual symptoms have implications on public approach to BED and to some extent clinical practice for the purpose of creating awareness in the public and for clinical practices.

\section{BED and psychiatric Morbidity}

The high psychiatric co-morbidity with BED found in our study is similar to findings in many other countries, mainly high income countries and also mainly from clinical and non-clinical populations cited under our Background, whether using DSM-IV or DSM-V criteria.

Our study therefore confirms the findings in our literature review (Background) that BED is associated with various psychiatric disorders. Our study reports an association not reported elsewhere, that is ED is associated with stress. However, our study did not explore the cause-effect relationship between different psychiatric disorders and BED.

\section{BED and Substance abuse.}


Our findings are similar to findings in High Income Countries on the association between BED and substance use disorder (SUD) whether using DSM-IV or DSM-V criteria for BED. However, direct comparison on the prevalence of SUD in BED is not possible because of the different methods used to determine SUD in BED and whether lifetime or current use. This could be the explanation for the finding in our study of an average of $3.1-7.6 \%$ which is lower than the lifetime reported prevalence of $30 \%-70 \%$ lifetime use in BED reported elsewhere in HICs and highlighted in our literature review, by Bellodi et al 2001 and Herpetz et all 2001 (Bellodi et al., 2001; Herpertz-Dahlmann et al., 2001). Our findings therefore concur with the findings in HIC that there is an association between SUD and BED. This difference between our findings and those from HICs could be that the drinking patterns are different in the different research participants.

Our study could not however establish the actual relationship between these associations - whether causal or casual and how the SUD is related to comorbidity with ED. Qualitative and biomarker studies are required to elucidate the nature of this association even in the Kenyan setting. Our findings suggest the need for routine screening of BED in clinical cases of BED and vice-versa.

Independent Indicators

It is noteworthy that no socio-demographic variable was associated with BED not even assumed age, given that high school students are younger than the rest. It is also noteworthy that wealth index of the family was not an independent predictor of BED. These findings suggest that BED occur in all sociodemographic variables reported in our study and also that BED occurs across the economic spectrum. The implication for this is BED is not a condition necessarily associated with affluence, at least in our study participants. Further, the fact that we found same prevalence in economically different countries with different cultural backgrounds would suggest cultural context has no bearing on expression of BED symptoms although levels of awareness may be different. Western studies cited in our literature have indicated that ED may go unnoticed even in clinical settings. Though both alcohol disorder and SUD were more in BED than in Non-ED, it was SUD that achieved significant levels. Most of the independent predictors were major depression and various types of anxiety disorders and increased stress levels. It is therefore not surprising that sedatives that are essentially anxiolytics were significantly associated with ED. These should be screened for and factored in any management of persons with ED.

Implications of our findings

There is need for public health awareness that BED is found in Kenya. The fact that we found a prevalence of $3.2 \%$ (and possibly $4.8 \%$ if we had used DSM-V criteria) in non-clinical student population means that the prevalence is higher in clinical population awareness at both clinical and community levels. This is necessary for clinicians, public health practitioners, family members and potential sufferers of ED. It is possible that students presenting with psychiatric disorders have existing BED that is not recognized and therefore not addressed. The youth may not volunteer this information unless prompted and even so may deny the existence of such a problem. A DSM-V based screener may be an easier and acceptable way to find out the existence of BED symptoms.

\section{Strengths of the study}

This study had a large sample of 9742 treatment naïve non clinical populations of students in four out of the 47 counties. The college and university students were from across the country because of the nationally centralized admissions to colleges and universities.

\section{Limitations of the study}

A major limitation of this study is that we used DSM-IV and not DSM-V leading to lesser prevalence. Because of the large sample it was not realistic to use researcher administered interview approaches. However, in population based surveys it is not uncommon to use self-administered screening instruments with the double edged risks for under and or over reporting. However, the use of DSM-IV had no effect on the various association.

Another main limitation is that this was a study on students as opposed to the general population. In mitigation, most youth in the communities belong either to the school, college and university going groups. They therefore represent the general population within the age groups studied. They also reported a significant proportion of the Kenyan population, given the pyramid population structured in Kenya. Although there were late age entry students, the contribution of age was determined at analysis level and found that age was not a predictive factor. A further limitation of our study was we did not include AN, which however is a lessor prevalent aspect of the overall Eating Disorder. However, our study with a wide age range is similar to other studies reported in the literature.

\section{Conclusion}

Subject to the limitations above, we have achieved our overall objective and the specific aims. In particular we have established the prevalence of BED, associated psychiatric conditions and SUD, the independent predictors of BED. On the basis of all these, we are able to suggest potential approach on possible interventions through public and service providers' awareness.

\section{Future Studies}

This study was essentially an exploratory cross-sectional study. However, our findings point out to areas that need further studies and in particular the nature of the association between ED, psychiatric disorders and SUD. Mixed methods i.e. qualitative and quantitative including biomarker and a longitudinal approach would determine the nature of the associations. Prevalence studies of AN and how they co-morbid with BED are recommended.

A peculiar aspect of this study is not so much what value it adds to studies in High Income Countries, but that LMIC's constitute the largest proportion in addition to greater burden of mental disorder of global population, and therefore on important contribution to the global data base. Further, the environment which varies greatly across different settings, interact with biological factors to determine development and behavioral outcomes, and therefore important to see how prevalence and associations compares between HIC and LMIC.

\section{Declarations}

Page 12/16 
Ethics approval and consent to participate:

Ethical approval was granted by the Maseno University Ethics Review Board in Kenya (IRB number MSU/DRPI/MUERC/00344/16).

\section{Informed consent:}

Informed consent was obtained from participants aged $18+$ and from parents of participants who were below the age of 18 . Participants below 18 years signed assent forms.

\section{Consent for publication:}

Not applicable

Competing interests:

None

\section{Guidelines}

All the guidelines have been followed and adhered to as per the journal requirements.

\section{Funding:}

Africa Mental Health Research and Training Foundation (AMHRTF) in-house support for this particular set of data collection and analysis. The Foundation did not influence researchers work independently.

\section{Authors' contributions:}

VNM - oversight on data collection; drafting of the paper; DMN - conceptualization and oversight of the study; drafting of the paper; ENM - literature review; RKdata analysis; LO-data analysis; FK- drafting ; MM- literature review ; CM - Ethical conduct of the study and data management; DM - conceptualization of the study.

\section{Acknowledgement}

All the participants who participated in the study and the different institutions and communities who facilitated the study, Africa Mental Health Research and Training Foundation for facilitating the study.

\section{References}

American Psychiatric Association. (2013). Diagnostic and Statistical Manual of Mental Disorders (5th ed.). Arlington VA: American Psychiatric Association.

Arcelus, J., Mitchell, A. J., Wales, J., \& Nielsen, S. (2011). Mortality rates in patients with anorexia nervosa and other eating disorders: a meta-analysis of 36 studies. Archives of General Psychiatry, 68(7), 724-731.

Bellodi, L., Cavallini, M. C., Bertelli, S., Chiapparino, D., Riboldi, C., \& Smeraldi, E. (2001). Morbidity risk for obsessive-compulsive spectrum disorders in firstdegree relatives of patients with eating disorders. American Journal of Psychiatry, 158(4), 563-569.

Brewerton, T. D. (2011). Posttraumatic stress disorder and disordered eating: food addiction as self-medication. Mary Ann Liebert, Inc. 140 Huguenot Street, 3rd Floor New Rochelle, NY 10801 USA.

Bulik, C. M., Klump, K. L., Thornton, L., Kaplan, A. S., Devlin, B., Fichter, M. M., ... Crow, S. (2004). Alcohol use disorder comorbidity in eating disorders: a multicenter study. The Journal of Clinical Psychiatry.

Campbell, D. T., \& Fiske, D. W. (1959). Convergent and discriminant validation by the multitrait-multimethod matrix. Psychological Bulletin, $56(2), 81$.

Cochrane, C., Malcolm, R., \& Brewerton, T. (1998). The role of weight control as a motivation for cocaine abuse. Addictive Behaviors, $23(2), 201-207$.

Cohen, L. R., Greenfield, S. F., Gordon, S., Killeen, T., Jiang, H., Zhang, Y., \& Hien, D. (2010). Survey of eating disorder symptoms among women in treatment for substance abuse. The American Journal on Addictions, 19(3), 245-251.

de Zwaan, M. (2001). Binge eating disorder and obesity. International Journal of Obesity, 25(1), S51-S55.

Fornaro, M., Daray, F. M., Hunter, F., Anastasia, A., Stubbs, B., De Berardis, D., ... Fusar-Poli, P. (2020). The prevalence, odds and predictors of lifespan comorbid eating disorder among people with a primary diagnosis of bipolar disorders, and vice-versa: systematic review and meta-analysis. Journal of Affective Disorders.

Garfinkel, P. E., Garner, D. M., Kaplan, A. S., Rodin, G., \& Kennedy, S. (1983). Differential diagnosis of emotional disorders that cause weight loss. Canadian Medical Association Journal, 129(9), 939. 
Grace, K., Davenport, F., Funk, C., \& Lerner, A. M. (2012). Child malnutrition and climate in Sub-Saharan Africa: An analysis of recent trends in Kenya. Applied Geography, 35(1-2), 405-413.

Hay, P., Girosi, F., \& Mond, J. (2015). Prevalence and sociodemographic correlates of DSM-5 eating disorders in the Australian population. Journal of Eating Disorders, 3(1), 1-7.

Herpertz-Dahlmann, B., Müller, B., Herpertz, S., Heussen, N., Hebebrand, J., \& Remschmidt, H. (2001). Prospective 10-year follow-up in adolescent anorexia nervosa-course, outcome, psychiatric comorbidity, and psychosocial adaptation. Journal of Child Psychology and Psychiatry, 42(5), 603-612.

Holderness, C. C., Brooks-Gunn, J., \& Warren, M. P. (1994). Co-morbidity of eating disorders and substance abuse review of the literature. International Journal of Eating Disorders, 16(1), 1-34.

Hsu, L. K. G., Kaye, W., \& Weltzin, T. (1993). Are the eating disorders related to obsessive compulsive disorder? International Journal of Eating Disorders, 14(3), 305-318.

Humeniuk, R., Henry-Edwards, S., Ali, R., Poznyak, V., Monteiro, M. G., \& World Health Organization. (2010). The Alcohol, Smoking and Substance involvement Screening Test (ASSIST): manual for use in primary care/prepared by R. HumeniukU [et al]. In The Alcohol, Smoking and Substance involvement Screening Test (ASSIST): manual for use in primary care/prepared by R. HumeniukU [et al].

Ivarsson, T., Råstam, M., Wentz, E., Gillberg, I. C., \& Gillberg, C. (2000). Depressive disorders in teenage-onset anorexia nervosa: a controlled longitudinal, partly community-based study. Comprehensive Psychiatry, 41(5), 398-403.

Iwasaki, Y., Matsunaga, H., Kiriike, N., Tanaka, H., \& Matsui, T. (2000). Comorbidity of axis I disorders among eating-disordered subjects in Japan. Comprehensive Psychiatry, 41(6), 454-460.

Jarry, J. L., \& Vaccarino, F. J. (1996). Eating disorder and obsessive-compulsive disorder: neurochemical and phenomenological commonalities. Journal of Psychiatry and Neuroscience, 21(1), 36.

Kenya National Bureau of Statistics (KNBS). (2019). 2019 KENYA POPULATION AND HOSUING CENSUS VOLUME IV:Distribution of Population by SocioEconomic Characteristics. Na. Retrieved from https://www.knbs.or.ke/?wpdmpro=2019-kenya-population-and-housing-census-volume-iv-distribution-ofpopulation-by-socio-economic-characteristics

Keski-Rahkonen, A., \& Mustelin, L. (2016). Epidemiology of eating disorders in Europe: prevalence, incidence, comorbidity, course, consequences, and risk factors. Current Opinion in Psychiatry, 29(6), 340-345.

Kessler, R. C., McGonagle, K. A., Zhao, S., Nelson, C. B., Hughes, M., Eshleman, S., ... Kendler, K. S. (1994). Lifetime and 12-month prevalence of DSM-III-R psychiatric disorders in the United States: results from the National Comorbidity Survey. Archives of General Psychiatry, 51(1), 8-19.

Koenigsberg, H. W., Kaplan, R. D., Gilmore, M. M., \& Cooper, A. M. (1985). The relationship between syndrome and personality disorder in DSM-III: experience with 2,462 patients. The American Journal of Psychiatry.

Krug, I., Treasure, J., Anderluh, M., Bellodi, L., Cellini, E., Di Bernardo, M., ... Penelo, E. (2008). Present and lifetime comorbidity of tobacco, alcohol and drug use in eating disorders: A European multicenter study. Drug and Alcohol Dependence, 97(1-2), 169-179.

Lewinsohn, P. M., Striegel-Moore, R. H., \& Seeley, J. R. (2000). Epidemiology and natural course of eating disorders in young women from adolescence to young adulthood. Journal of the American Academy of Child \& Adolescent Psychiatry, 39(10), 1284-1292.

Lilenfeld, L. R., Kaye, W. H., Greeno, C. G., Merikangas, K. R., Plotnicov, K., Pollice, C., ... Nagy, L. (1997). Psychiatric disorders in women with bulimia nervosa and their first-degree relatives: Effects of comorbid substance dependence. International Journal of Eating Disorders, 22(3), $253-264$.

Lipson, S. K., \& Sonneville, K. R. (2020). Understanding suicide risk and eating disorders in college student populations: Results from a National Study. International Journal of Eating Disorders, 53(2), 229-238.

Mamah, D., Owoso, A., Sheffield, J. M., \& Bayer, C. (2014). The WERCAP screen and the WERC stress screen: psychometrics of self-rated instruments for assessing bipolar and psychotic disorder risk and perceived stress burden. Comprehensive Psychiatry, 55(7), 1757-1771.

Mandelli, L., Draghetti, S., Albert, U., De Ronchi, D., \& Atti, A.-R. (2020). Rates of comorbid Obsessive-Compulsive Disorder in Eating Disorders: a meta-analysis of the literature. Journal of Affective Disorders, 12404.

McDonald, C. E., Rossell, S. L., \& Phillipou, A. (2019). The comorbidity of eating disorders in bipolar disorder and associated clinical correlates characterised by emotion dysregulation and impulsivity: A systematic review. Journal of Affective Disorders, 259, 228-243.

McElroy, S. L., Phillips, K. A., \& Keck Jr, P. E. (1994). Obsessive compulsive spectrum disorder. The Journal of Clinical Psychiatry, $55,33-51$.

Meier, M., Kossakowski, J. J., Jones, P. J., Kay, B., Riemann, B. C., \& McNally, R. J. (2020). Obsessive-compulsive symptoms in eating disorders: A network investigation. International Journal of Eating Disorders, 53(3), 362-371. 
Mezzich, J. E., Fabrega, H., Coffman, G. A., \& Haley, R. (1989). DSM-III disorders in a large sample of psychiatric patients: frequency and specificity of diagnoses. The American Journal of Psychiatry.

Miotto, P., Pollini, B., Restaneo, A., Favaretto, G., Sisti, D., Rocchi, M. B. L., \& Preti, A. (2010). Symptoms of psychosis in anorexia and bulimia nervosa. Psychiatry Research, 175(3), 237-243.

Naghavi, M., Abajobir, A. A., Abbafati, C., Abbas, K. M., Abd-Allah, F., Abera, S. F., ... Murray, C. J. L. (2017). Global, regional, and national age-sex specifc mortality for 264 causes of death, 1980-2016: A systematic analysis for the Global Burden of Disease Study 2016. The Lancet, 390(10100).

https://doi.org/10.1016/S0140-6736(17)32152-9

Ndetei, D. M., Khasakhala, L. I., Mutiso, V., Ongecha-Owuor, F. A., \& Kokonya, D. A. (2009). Patterns of drug abuse in public secondary schools in Kenya. Substance Abuse, 30(1), 69-78.

Ndetei, D. M., Khasakhala, L. I., Mutiso, V., Ongecha-Owuor, F. A., \& Kokonya, D. A. (2010). Drug use in a rural secondary school in Kenya. Substance Abuse, 31(3), $170-173$.

Reba-Harrelson, L., Von Holle, A., Hamer, R. M., Swann, R., Reyes, M. L., \& Bulik, C. M. (2009). Patterns and prevalence of disordered eating and weight control behaviors in women ages 25-45. Eating and Weight Disorders-Studies on Anorexia, Bulimia and Obesity, 14(4), e190-e198.

Rijkers, C., Schoorl, M., van Hoeken, D., \& Hoek, H. W. (2019). Eating disorders and posttraumatic stress disorder. Current Opinion in Psychiatry, $32(6), 510-517$.

Robins, L. N. (1991). Psychiatric disorders in America. The Epidemiologic Catchment Area Study.

Schwalberg, M. D., Barlow, D. H., Alger, S. A., \& Howard, L. J. (1992). Comparison of bulimics, obese binge eaters, social phobics, and individuals with panic disorder on comorbidity across DSM-III-R anxiety disorders. Journal of Abnormal Psychology, 101(4), 675.

Shear, M. K., Greeno, C., Kang, J., Ludewig, D., Frank, E., Swartz, H. A., \& Hanekamp, M. (2000). Diagnosis of nonpsychotic patients in community clinics. American Journal of Psychiatry, 157(4), 581-587.

Smits, J., \& Steendijk, R. (2015). The international wealth index (IWI). Social Indicators Research, 122(1), 65-85.

Specker, S., de Zwaan, M., Raymond, N., \& Mitchell, J. (1994). Psychopathology in subgroups of obese women with and without binge eating disorder. Comprehensive Psychiatry, 35(3), 185-190.

Sullivan, P. F., Bulik, C. M., Fear, J. L., \& Pickering, A. (1998). Outcome of anorexia nervosa: a case-control study. American Journal of Psychiatry, 155(7), 939946 .

Swanson, S. A., Crow, S. J., Le Grange, D., Swendsen, J., \& Merikangas, K. R. (2011). Prevalence and correlates of eating disorders in adolescents: Results from the national comorbidity survey replication adolescent supplement. Archives of General Psychiatry, 68(7), 714-723.

Udo, T., \& Grilo, C. M. (2019). Psychiatric and medical correlates of DSM-5 eating disorders in a nationally representative sample of adults in the United States. International Journal of Eating Disorders, 52(1), 42-50.

Vastag, B. (2001). What's the connection? No easy answers for people with eating disorders and drug abuse. Jama, 285(8), $1006-1007$.

Wilfley, D. E., Friedman, M. A., Dounchis, J. Z., Stein, R. I., Welch, R. R., \& Ball, S. A. (2000). Comorbid psychopathology in binge eating disorder: Relation to eating disorder severity at baseline and following treatment. Journal of Consulting and Clinical Psychology, 68(4), 641.

Wolfe, W. L., \& Maisto, S. A. (2000). The relationship between eating disorders and substance use: moving beyond co-prevalence research. Clinical Psychology Review, 20(5), 617-631.

Woodside, D. B., Garfinkel, P. E., Lin, E., Goering, P., Kaplan, A. S., Goldbloom, D. S., \& Kennedy, S. H. (2001). Comparisons of men with full or partial eating disorders, men without eating disorders, and women with eating disorders in the community. American Journal of Psychiatry, 158(4), 570-574.

World Health Organization. (2010). mhGAP intervention guide - For mental, neurological and substance abuse disorders in non-specialized health settings: Mental Health Gap Action Programme. World Health Organization. Retrieved from http://scholar.google.com/scholar?

$\mathrm{hl}=$ en\&btnG=Search\&q=intitle:mhGAP+Intervention+Guide\# $1 \% 5 \mathrm{Cnhttp}: / /$ scholar.google.com/scholar?

$\mathrm{hl}=\mathrm{en} \& \mathrm{btn} \mathrm{G}=$ Search\&q=intitle:mhGAP+intervention+guide\#1

Zimmerman, M., \& Mattia, J. I. (2001). The Psychiatric Diagnostic Screening Questionnaire: development, reliability and validity. Comprehensive Psychiatry.

\section{Figures}




\section{Eating Disorders Items \\ $\mathrm{N}=9742$}

Did you often go on eating binges (eating a very large amount of food very quickly over a short period of time)?

To prevent gaining weight from an eating binge did you go on strict diets or exercise excessively?

Did you often feel you could not control how much you were eating during an eating binge?

Was your weight, or the shape of your body, one of the most important things that affected your opinion of yourself?

Did you go on eating binges during which you ate so much that you felt uncomfortably full?

Did you go on eating binges during which you ate a large amount of food even when you didnt feel hungry?

Did you go on eating binges and then feel disgusted with yourself afterwards?

Were you very upset with yourself because you were going on eating

$$
\text { binges? }
$$

Did you eat alone during an eating binge because you were embarrassed by how much you were eating?

To prevent weight gain from an eating binge did you force yourself to vomit or use laxatives or water pills?
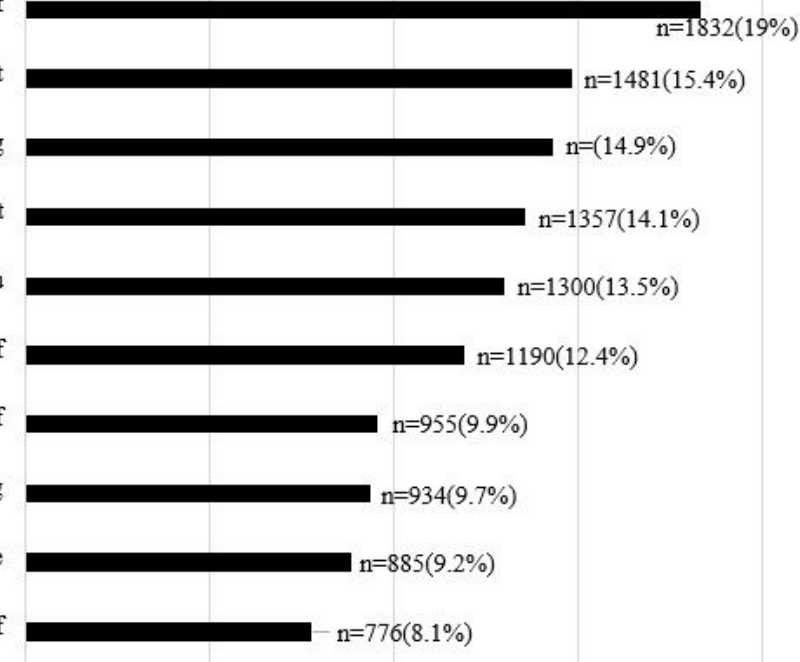

$\begin{array}{llll}0 & 500 & 1000 & 1500\end{array}$

\section{Figure 1}

Prevalence of symptoms of Bulimia/Binge Eating Disorder 